Document downloaded from:

http://hdl.handle.net/10251/65816

This paper must be cited as:

Ortega Higueruelo, FJ.; Bañuls Polo, M.; Sanza, FJ.; Laguna, MF.; Holgado Bolaños, M.; Casquel Del Campo, R.; Angulo Barrios, C.... (2013). Development of a versatile biotinylated material based on SU-8. Journal of Materials Chemistry B. 1:2750-2756. doi:10.1039/c3tb20323a.

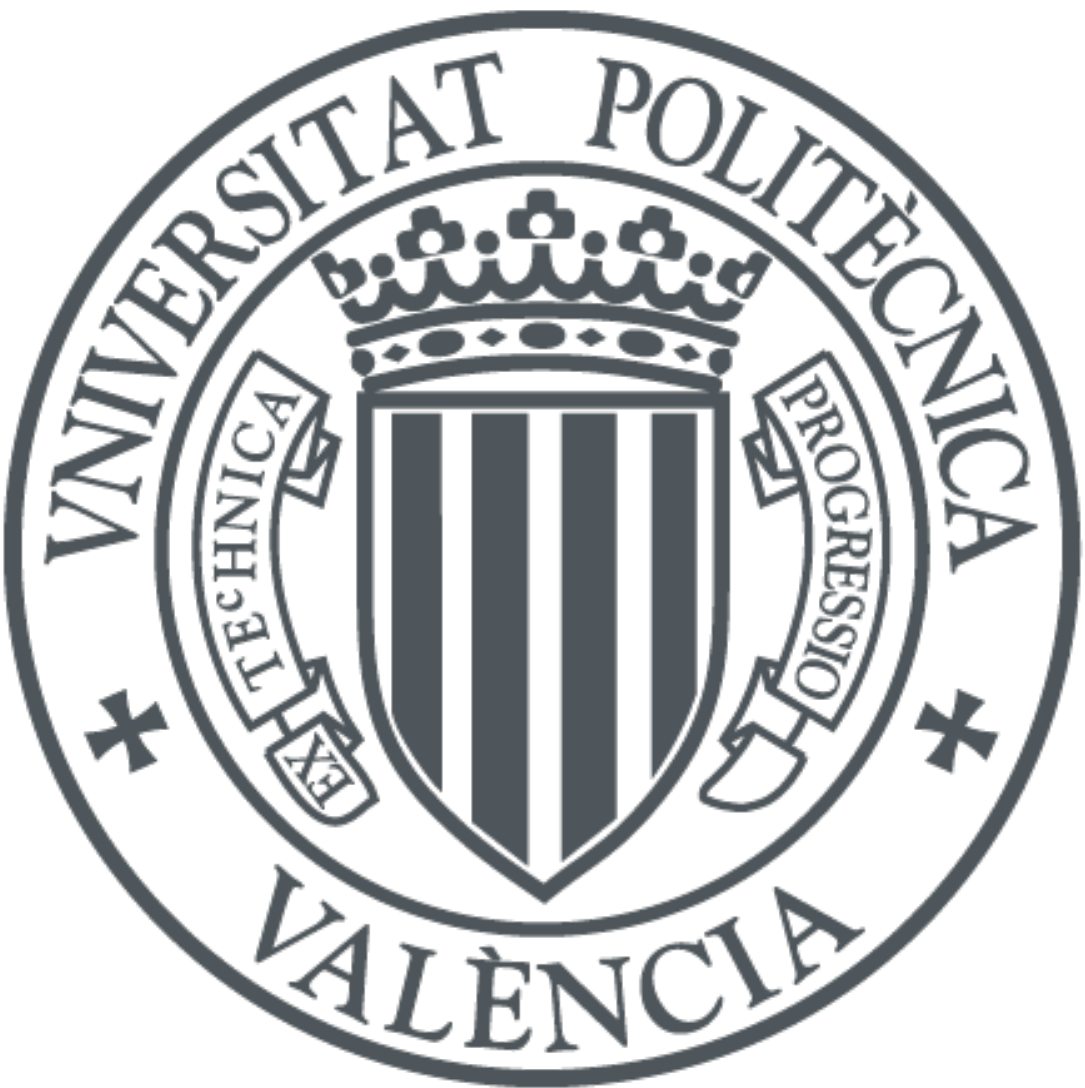

The final publication is available at

https://dx.doi.org/10.1039/c3tb20323a

Copyright Royal Society of Chemistry

Additional Information 


\title{
Development of a versatile biotinylated material based on $\mathrm{SU-8}$
}

\author{
Francisco J. Ortega ${ }^{a}$, María-José Bañuls ${ }^{a}$, Francisco J. Sanza ${ }^{b}$, María F. Laguna ${ }^{b}$, Miguel Holgado $^{b}$, \\ Rafael Casquel $^{b}$, Carlos A. Barrios ${ }^{c}$, David López-Romero ${ }^{c}$, Ángel Maquieira ${ }^{a}$ and Rosa Puchades ${ }^{* a}$
}

The negative epoxy-based SU-8 photoresist has a wide variety of applications within semiconductors industry, photonics and lab-on-a-chip devices, and it is emerging for sensing purposes to replace siliconbased devices. In the present work, biotinylation of SU-8 polymer surface promoted by light is reported. As a result, a novel, effective, and low-cost material focused to the immobilization of bioreceptors and 10 consequent biosensing is developed. This material allows the spatial discrimination depending on the irradiation of desired areas. The most salient feature is that the photobiotin may be directly incorporated to the SU-8 curing process, reducing consequently times and costs. The potential use of this substrate is demonstrated by the immunoanalytical detection of the synthetic steroid gestrinone, showing excellent performances. Moreover, the naked eye biodetection due to the transparent SU-8 substrate or simple 15 instrumental quantification are additional advantages.

\section{Introduction}

Optical biosensors are powerful analytical tools with application in healthcare, biomedical and pharmaceutical research, environmental monitoring, and security, among others. ${ }^{1}$ ${ }_{20}$ Commonly used materials for production of miniaturized sensors include silicon, silicon oxide, silicon nitride, and gold. These devices are made using standard microfabrication techniques, such as oxidation and chemical or physical vapor deposition, lithography, etc. This adds complexity, cost and production time 25 to sensor fabrication. Polymeric materials are an alternative to silicon derivatives because of their low-cost, high-throughput production methods, ${ }^{2}$ and greater chance of chemical functionalization on demand.

The SU-8 polymer (glycidyl ether of bisphenol A) is an epoxy30 based negative photoresist with excellent mechanical, physical, and optical properties after polymerization. ${ }^{3} \mathrm{SU}-8$ is a valuable material used in a wide range of applications such as optical waveguides for telecommunication; ${ }^{4}$ microelectromechanical systems (MEMS) $)^{5,6}$ including microfluidic structures, ${ }^{7,8}$ probes 35 for microscopy ${ }^{9,10}$ and molds for microembossing; ${ }^{11,12}$ and microfabrication-based sensors. ${ }^{13,14}$

Due to its chemical functionalization capability on demand and low cost, SU-8 is an attractive substrate candidate for the fabrication of bioanalytical micro and nano-devices. Although its 40 surface hydrophobicity is a limitation, causing the high nonspecific adsorption of probes and targets, and dificulting the surface wettability, ${ }^{15}$ these drawbacks can be resolved by chemically tailoring the SU-8 surface properties.

When SU-8 is used for biosensing, probe immobilization can 45 be done by covalent, electrostatic, or adsorptive interactions. SU8 chemical surface modification can be accomplished to include at least one functional group, such as $\mathrm{CHO}, \mathrm{NH}_{2}$, or $\mathrm{SH}$, which can be used for covalent binding biologically active probes, ${ }^{16-20}$ or for solid phase synthesis.. ${ }^{21,22}$ Direct covalent immobilization 50 of aminated or thiolated moieties is possible as SU-8 epoxide rings can undergo $\mathrm{S}_{\mathrm{N}} 2$ reactions.

The functionalization of SU-8 for biosensing is an upcoming field. Thus, the direct immobilization of aminoalkyl, thiophosphoryl and phosphonylated single strand DNA on SU-8 55 to set up microarray based hybridization assays is reported. ${ }^{16,23}$ Blagoi's group described the SU-8 polymer treatment with 3aminopropyltriethoxysilane and glutaraldehyde -this procedure is similar to the well known organosilane based silicon derivatization- to detect C-reactive protein by fluorescent 60 sandwich immunoassay on SU-8 microwells. ${ }^{17}$ Using a similar strategy, Joshi et al. immobilized human immunoglobulins on microfabricated SU-8 cantilevers although none immunoassay demonstration was reported. ${ }^{18}$ In other work, Joshi et al. performed SU-8 microcantilevers surface modification by 65 grafting amine groups using pyrolytic dissociation of ammonia in a hotwire CVD setup; after glutaraldehyde treatment, human immunoglobulin was attached to the surface and recognized by a fluorescent labeled anti-human antibody ${ }^{19}$ Using the same immunoreagents, Deepu et al. employed glycine and 1170 mercaptoundecanoic acid to covalently attach the human immunoglobulins using carbodiimide bioconjugation chemistry. ${ }^{20}$ Also, SU-8 encoded microparticles are chemicaly modified to attach oligonucleotides or proteins to perform multiplexed assays. ${ }^{24,25}$. Recenlty, a swelling-deswelling method for the facile 75 surface modification of $2 \mathrm{D}$ and $3 \mathrm{D}$ patterns of SU-8 has been described, demonstrating the oligonucleotide attachment. ${ }^{26}$ However, this method involves the use of toluene, and this makes it unapplicable to the attachment of proteins due to the risk of denaturation. Regarding the SU-8 surface modification of 80 microfabricated structures for label-free optical biosensing, there are few examples in the literature, and they are based on adsorption or covalent attachment after the fabrication of the microstructures. ${ }^{13,27-31}$

The bioreceptor spatial localization on a surface is a powerful 85 approach to generate molecular arrays for analytical or bioelectronic applications. In this sense, photoactivation of lightsensitive reagents on a solid surface through a photomask allows the generation of reactive intermediates, such as radicals, carbenes, and nitrenes, which react with the surface yielding 90 coated domains of the reactive intermediates. ${ }^{32}$ Until now, these approaches have been applied in materials like polystyrene, nitrocellulose, and silicon, but scarcely with SU-8. ${ }^{15,33}$ 
The derivative N-(4-azido-2-nitrophenyl)-N'-(3biotinylaminopropyl)-N'-methyl-1,3-propanediamine, commonly called photobiotin ( $\mathrm{Phb}$ ), is a particularly suitable compound belonging to the generation of extremely photoreactive species. 5 The specific binding of biotin to streptavidin presents a very high affinity $\left(\mathrm{K}_{\mathrm{a}}=10^{15} \mathrm{M}^{-1}\right),{ }^{34}$ and its tetravalency allows for a simultaneous binding to several biotin emerging from the surface via a biotin/streptavidin/biotin bridge. ${ }^{35,36}$ Upon UV irradiation $(\lambda$ $=350 \mathrm{~nm}$ ), the photoactivatable group of Phb gives rise to a 10 nitrene from an azide, which can be inserted readily into both $\mathrm{C}$ $\mathrm{H}$ as well as other functional groups, such as $\mathrm{OH}, \mathrm{NH}, \mathrm{NO}_{2}, \mathrm{C}=\mathrm{C}$, etc. $^{37}$ This is a well established methodology for protein biotinylation.

In this paper, we propose a biotin-modified SU-8 material 15 providing a generic biosurface for the effective immobilization of any biotinylated molecule, via a streptavidin intermediate. To this end, the SU-8 surface can be coated with photobiotin and irradiated through a photomask to yield the patterned surface. Also, we demonstrate the biotinylation during the SU-8 20 fabrication process as the wavelenght used to attach the Phb overlap with the wavelenght needed for the croslinking of the photoresist.

The main advantage of the proposed method against the approaches already described in the literature ${ }^{16-20,23,26-31}$ for the 25 biofunctionalization of SU-8 include the spatial discrimination obtained by irradiating only on the desired areas-, the suitability to be incorporated within the SU-8 polymer standard fabrication protocol -thus saving time and cost-, and the versatility of the surface obtained, as any biotinylated receptor can be attached to 30 the surface by an streptavidine bridge.

\section{Experimental}

\section{Chemicals}

SU-8 polymer and SU-8 developer are distributed through MicroChem Corp. (Newton, MA, USA) in several formats 35 depending on their composition. In this paper, SU-8 2000.5 was employed attending to the desired layer thickness. Streptavidin, streptavidin-gold ( 2.5 A520 units/mL), streptavidin-ATTO 655 from Streptomyces avidinii, gold-labeled goat anti-rabbit immunoglobulin (GAR-Au), anti-bovine albumin antibody 40 produced in rabbit (a-BSA), silver enhancer solutions A and B, ovalbumin (OVA) lyophilized powder, photobiotin (Phb), mercaptoethanol, ethanolamine, hydrogen peroxide $35 \% \mathrm{w} / \mathrm{w}$, and sulfuric acid 95-98\% were purchased from Sigma-Aldrich Química (Madrid, Spain). Anti-biotin polyclonal antibody 45 produced in rabbit (a-biotin) was provided by Abcam (Cambridge, United Kingdom). Dimethylsulfoxide (DMSO) was acquired from Acros Organics (Madrid, Spain). PBS (10 mmol/L sodium phosphate, $137 \mathrm{mmol} / \mathrm{L} \mathrm{NaCl}, 2.7 \mathrm{mmol} / \mathrm{L} \mathrm{KCl}, \mathrm{pH} 7.4$ ) and PBS-T (PBS containing $0.05 \%$ Tween 20) were used as 50 buffers. The anti-gestrinone polyclonal antibody and the gestrinone hapten cojugate were obtained by our research group. ${ }^{38}$

\section{Instruments}

${ }_{55}$ The spin-coating process was performed with a WS-400BX6MPP/LITE spin-coater (Laurell Technologies Corp., North
Wales, PA, USA). Microarray printing was carried out with a low volume non-contact dispensing system from Biodot (Irvine, CA, USA), model AD1500. SU-8 and SU-8/photobiotin curing was ${ }_{60}$ done with a laser workstation from Optec, Inc. (Lowell, MI, USA), model ML-100, using a high power diode-pumped solid state (DPSS) Q-switched laser of Nd:YVO4 from Spectra Physics (Irvine, CA, USA), model HIPPO 355-5, with output at $350 \mathrm{~nm}$ wavelength. Glass slides were purchased from Menzel-Gläser ${ }_{65}$ (Braunschweig, Germany). UV-Vis spectra measurements were obtained with a spectrophotometer from Agilent Technologies (Santa Clara, CA, USA), model 8453. The hot-plate model Agimatic E-C was acquired from JP-Selecta (Barcelona, Spain). Signal measure of Ag complex was carried out with a document 70 scanner from Seiko Epson Corp. (Nagano, Japan), model EU-34, and images were processed with Adobe Photoshop CS software from Adobe Systems Inc. (San Jose, CA, USA). For standard microarray image analysis, GenePix Pro 6.0 software from Molecular Devices, Inc. (Sunnyvale, CA, USA) was employed. ${ }_{75}$ Contact angle system OCA20 equipped with SCA20 software was from Dataphysics Instruments GmbH (Filderstadt, Germany). A Bruker Tensor 27 equipped with an ATR dura SamplIR accessory was used to acquire FTIR surface spectra.

\section{${ }_{80}$ Deposition of SU-8 and SU-8/Phb layers on glass slides}

In order to obtain maximum process reliability, the substrate was clean and dry prior to applying SU-8 2000.5 resist. First, a glass chip of $1 \mathrm{~mm}$ of thickness was treated with piranha solution $\left(\mathrm{H}_{2} \mathrm{O}_{2}: \mathrm{H}_{2} \mathrm{SO}_{4}\right)$ wet etching, followed by de-ionized water (DI)${ }_{85} \mathrm{H}_{2} \mathrm{O}$ and ethanol rinses and drying with $\mathrm{N}_{2}$. After a preheating process at $80{ }^{\circ} \mathrm{C}$ for $1 \mathrm{~min}$ applied to the chip, the SU-8 or SU$8 / \mathrm{Phb}$ mixture in the appropriate volumetric proportion was dispensed ( $1 \mathrm{~mL}$ resist $/ 5 \mathrm{~cm}^{2}$ substrate). To prepare the mixture, $\mathrm{Phb}$ was previously dissolved in DMSO at $100 \mu \mathrm{g} / \mathrm{mL}$. The 90 sample was spun at $4000 \mathrm{rpm}$ for 50 seconds, and soft baked at $80{ }^{\circ} \mathrm{C}$ for $1 \mathrm{~min}$ in a hotplate with good thermal control and uniformity. Next it was exposed to the DPSS laser radiation at $350 \mathrm{~nm}$ wavelength, defocused to $55 \%$ of maximum power, for $2 \mathrm{~min}$, baked at $80{ }^{\circ} \mathrm{C}$ for $1 \mathrm{~min}$, and dried with $\mathrm{N}_{2}$. Finally, the 95 glass slides were cut into suitable sizes with a tungsten awl.

\section{Photobiotin SU-8 coating}

SU-8 chip surface was biotinylated after fabrication as follows: $20 \mu \mathrm{L}$ of $\mathrm{Phb}(100 \mu \mathrm{g} / \mathrm{mL})$ in water were dispensed on the surface 100 of a chip and spread out using a coverslip. After $2 \mathrm{~h}$ in darkness, the surface was blown with $\mathrm{N}_{2}$. The chip was irradiated at $350 \mathrm{~nm}$ wavelength with a UV lamp for $20 \mathrm{~min}$, rinsed with (DI)- $\mathrm{H}_{2} \mathrm{O}$ and dried with $\mathrm{N}_{2}$ again.

The procedure to perform the patterning was the same but using 105 the adequate photomask instead of a coverslip.

\section{Anti-biotin immunoassay development}

Different concentrations of anti-BSA and anti-biotin antibodies, ranging from $1 \mu \mathrm{g} / \mathrm{mL}$ to $10 \mu \mathrm{g} / \mathrm{mL}$, in PBS-T buffer $(40 \mathrm{~nL})$, 110 were printed with a non contact automatic arrayer on SU-8 or $\mathrm{SU}-8 / \mathrm{Phb}$ surfaces. After incubation for $20 \mathrm{~min}$ at room temperature in a wet and dark environment, the samples were 
rinsed with PBS-T and (DI)- $\mathrm{H}_{2} \mathrm{O}$, and blown with $\mathrm{N}_{2}$. Then, 20 $\mu \mathrm{L}$ of OVA $1 \%$ in PBS were spread out on the microarray and incubated for $1 \mathrm{~h}$ as before. After washing with PBS-T and (DI)$\mathrm{H}_{2} \mathrm{O}$, the microarrays were incubated with a gold-labeled 5 secondary antibody (GAR-Au, $25 \mu \mathrm{g} / \mathrm{mL}$ in PBS-T) for $5 \mathrm{~min}$, followed by washing and drying as before. A mixture of $10 \mu \mathrm{L}$ of silver enhancer solution type A and $10 \mu \mathrm{L}$ of silver enhancer solution type B was added onto the microarray and spread out with a coverslip. After $20 \mathrm{~min}$, the chips were washed and dried.

${ }_{10}$ To quantify microarray signals, a high definition color image of the chip was acquired by an Epson EU-34 office scanner. The image was transformed to grey scale and 16-bit format for its data treatment with GenePix software.

\section{${ }_{15}$ Conjugation of streptavidin to gestrinone hapten}

The gestrinone oxime hapten 18 $\alpha$-Homo-pregna-4,9,11-trien$17 \beta$-ol-20-yn-3-carboxymethyl oxime $(\mathrm{GH})$ and the streptavidinGH conjugate were synthesized and purified as previously described. ${ }^{38}$ The concentration of streptavidin-GH conjugate and 20 the streptavidin/GH molecular ratio (3) was done by UV-Vis spectrophotometry.

\section{Gestrinone immunoassay development}

The biotinylated surface was treated with a solution of 25 streptavidin-GH $(991 \mu \mathrm{g} / \mathrm{mL}$ in PBS-T) for $20 \mathrm{~min}$. The chip was washed with PBS-T and (DI)- $\mathrm{H}_{2} \mathrm{O}$, rinsed with $\mathrm{N}_{2}$, and the surface blocked with OVA $1 \%$ in PBS-T for $1 \mathrm{~h}$. Then, $20 \mu \mathrm{L}$ of rabbit serum (dilution 1/4000 in PBS-T) and $20 \mu \mathrm{L}$ of gestrinone at different concentrations $(0.2,2,10$, and $20 \mathrm{ng} / \mathrm{mL})$ were mixed 30 and printed as described above, including a control blank. After incubation for $15 \mathrm{~min}$, the surface was washed and dried. Finally, $\mathrm{GAR}-\mathrm{Au}$ incubation, silver development and microarray quantification were performed as described above.

\section{${ }_{35}$ SU-8 and SU-8/Phb nanopillars fabrication}

Structures based on arrays of nanopillars were fabricated using SU-8 or a mixture of SU-8 and Phb $(100 \mu \mathrm{g} / \mathrm{mL}$ in DMSO) in a $1 / 1$ volumetric ratio. In both cases, the procedure was that previously described..$^{28,29}$

\section{${ }_{40}$ Results and discussion}

For the biotinylation of SU-8, the epoxy moieties could be used for covalent binding of amino groups like amino-biotin, but this method would not lead the spatial selectivity provided by photoactivation.

${ }_{45}$ As far as we know, there are only two reported SU-8 surface functionalization approaches allowing this spatial selectivity that can be achieved by using photoactivation. The approach described by Bagloi et al., 33 which consists in the micropatterning of SU-8 surfaces with antraquinone derivatives 50 and further covalent attachment of aminated biotin or other proteins; and the micropatterning approach developed by Wang et al. ${ }^{15}$ based on UV mediated graft polymerization of an acrylic acid derivative. However, none of them is demonstrated for biosensing.

${ }_{55} \mathrm{Phb}$ has been widely used in the literature for surface patterning of glass, silicon, and PDMS mainly. The mechanism of insertion occurs through a nitrene radical, allowing the insertion on $\mathrm{OH}, \mathrm{NH}$ and $\mathrm{CH}$ bonds. Thus, the biotinylation of SU-8 can take place as it is represented in figure 1 . However, it is 60 demonstrated that Phb has a considerable affinity towards the insertion into $\mathrm{C}-\mathrm{H}$ bonds and this must be the prefered insertion site.

65
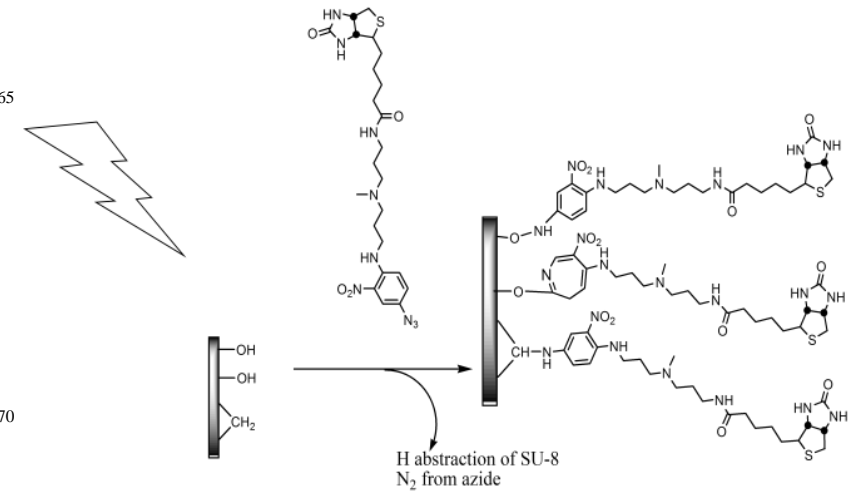

Fig. 1 Photobiotin insertion on SU-8 polymer under $350 \mathrm{~nm}$ light irradiation.

75

To proceed with the Phb tethering, it was necessary a previous step to open the SU-8 epoxy moieties transforming them into hydroxyl groups, this forbids the bioreceptor attachement through its amine groups. On the other hand, this change on the surface 80 increases its hydrophilicity, minimizing the protein adsorption and improving the surface wettability. For the opening procedure, an acidic pre-treatment of the surface was performed, and a buffer containing surfactant (Tween 20) was added to the protein solutions in order to prevent non-specific adsorption.

85 Attending to that, the epoxy-rings opening was done through acidic conditions, which were applied by immersing the chips in $1 \mathrm{M} \mathrm{H}_{2} \mathrm{SO}_{4}$ for one minute. After that, the insertion of biotin was done by spreading out an aqueous solution of Phb $100 \mu \mathrm{g} / \mathrm{mL}$ over the chip surface, and irradiating for $20 \mathrm{~min}$ at $350 \mathrm{~nm}$. In 90 order to assess the photobiotin immobilization, microarrays including several streptavidin-Au and GAR-Au concentrations were printed onto the biotinylated chips, with or without previous cleavage of epoxy rings using $1 \mathrm{M} \mathrm{H}_{2} \mathrm{SO}_{4}$. After incubation and further washings, the arrays were developed with the silver 95 reagent, showing a black precipitate when gold labeled protein is present on the surface. Results could be naked eye observed because the substrate is transparent, while quantitative monitoring was accomplished with a documental scanner.

Qualitative results are presented in Figure S1 (Supp. Material), 100 showing that photobiotin covalent immobilization on SU-8 chain is conditioned to the exposure with UV radiation and remaining on the surface only the streptavidin, without non-specific adsorption. With and without previous epoxy hydrolysis, a specific immobilization of streptavidin was observed, while GAR 105 was not retained on the surface. This fact indicated the success in the surface selective biotin photoattachment. When UV irradiation was avoided (after photobiotin surface application) no signal was observed nor with streptavidin neither with GAR if 
previous epoxy acidic hydrolysis was performed. When no epoxy hydrolysis was carried out, both streptavidin and GAR resulted immobilized on the surface. This may be explained attending to the covalent attachment of both proteins to the surface by means 5 of a nucleophilic attack from protein amine groups to the epoxy moieties. It is in agreement with that found in the literature, where different authors use the epoxy hydrolysis to reduce or avoid the unwanted protein immobilization on SU-8 surface, or demonstrate the covalent nature of the protein attachment, using 10 cerium ammonium nitrate, ${ }^{17}$ sulfochromic solution, ${ }^{13,18}$ chrome etch, ${ }^{16}$ or PEG grafting. ${ }^{15}$

The surface characterization of biotin modified chips was performed using X-ray photoelectron spectroscopy (XPS) and contact angle measurements. Results showed the presence of $S$ 15 and $\mathrm{N}$ atoms in the treated chips, whereas these chemical elements were not found in bare SU-8 chips. Contact angle values showed an expected decrease associated with an increase in the surface hydrophilic character, due to the acidic ring opening and further biotin incorporation. For SU-8 chips, the contact angle 20 value was $82^{\circ}$, whereas the value was $69^{\circ}$ for the hydrolyzed SU8 chip, and $67^{\circ}$ for the biotinylated polymer (Figure 2).

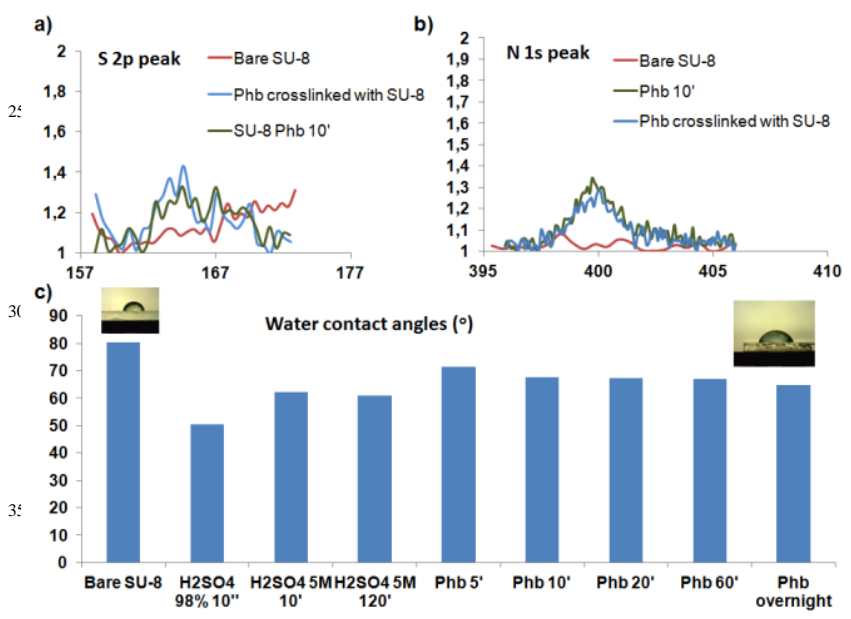

Fig. 2 XPS analysis (a) S 2p peak and (b) N 1s peak for bare SU${ }_{40} 8$ surface, SU-8 polymer coated with photobiotin, and biotinylated SU-8 surface employing SU-8 and photobiotin polymerization mixture. (c) Contact angle values for both SU-8, bare and under different hydrolysis and biotinylation conditions.

45 In order to ensure the availability of this new surface to develop immunoassays, immunoreaction between biotin and a specific antibiotin polyclonal antibody was studied. For that, two microarrays (dimension $3 \times 3$ ) were printed on the biotinylated material, one of a-BSA antibody as a control, and the other one 50 using the specific antibody a-biotin. A gold labelled secondary antibody (GAR-Au) was added to detect signals derived from biotin/a-biotin interaction. To avoid GAR-Au non-specific adsorption, several blocking agents such as OVA, ethanolamine, and mercaptoethanol were tested. OVA provided the best results 55 probably because mercaptoethanol and ethanolamine interact with photobiotin to form intermolecular H-bonds. Also, the blocking step was assayed before and after the primary antibody incubation step. Blocking after the incubation with primnary antibody provided much better results in terms of specificity (Fig. ${ }_{60} 3$ ). This effect may be explained by the OVA size, which is larger than photobiotin, so the coating totally loses its specificity. It can be observed through similar intensity for a-biotin and a-BSA microarrays. Even without OVA blocking, the immunoreaction was possible; hence immobilisation and recognition steps were ${ }_{65}$ performed in conditions (using an appropriate surfactant in the buffer) so that non-specific adsorption was almost eliminated. In any case, the use of OVA blocking helped to reduce the background signal.

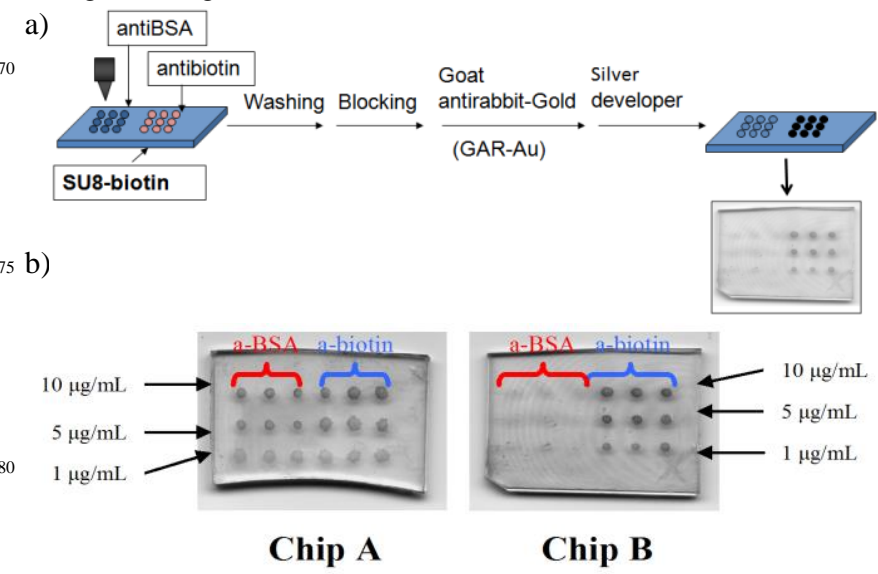

Fig. 3 (a) Scheme of the immunoassay designed to evaluate the 85 bioavailability of the biotin immobilized on the biotinylated SU8. (b) Image of the resulted microarray, chip A is not coated with photobiotin and chip B has a photobiotinylated surface.

To prove the capability of the developed approach for surface 90 patterning, Phb $100 \mu \mathrm{g} / \mathrm{mL}$ was deposited over hydrolyzed SU-8 surface. The irradiation at $350 \mathrm{~nm}$ was performed during $10 \mathrm{~min}$ through a photomask as it is represented in figure 4. After washings, streptavidin-ATTO $65550 \mu \mathrm{g} / \mathrm{mL}$ in PBS-T was incubated over the surface for $5 \mathrm{~min}$ at room temperature, and 95 was washed with PBS-T and water. The fluorescence was registered showing a patterned surface.

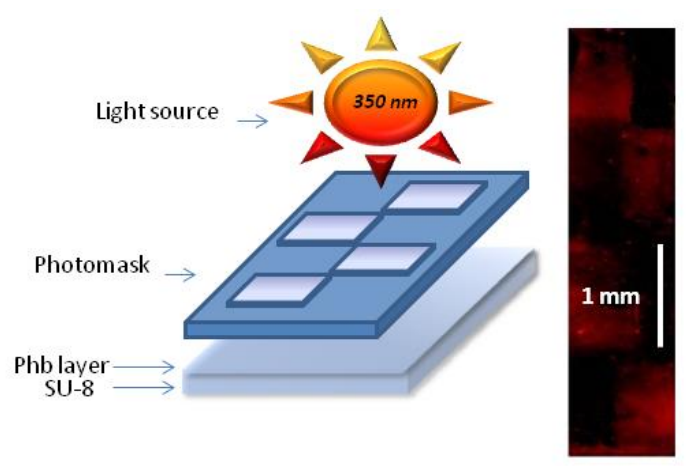

Fig 4. Fluorescence image obtained after irradiation through a photomask

Once demonstrated the effectivity of the procedure for the 115 selective SU-8 biotinylation maintaining the bioavailability, the following step was to analyze the photobiotinylation of SU-8 in 
the UV crosslinking step carried out during the polymer fabrication process. The inclussion of additives, such as nanoparticles in the SU-8 to modify its electrical (or magnetical) properties maintaining its photopatterning behaviour has been 5 already demonstrated. ${ }^{39}$ Thus, the biotinylation of the material simultaneously to the photoresist polymerization proccess is highly interesting, specially for the construction of label-free nanobiosensing devices, as it offers the possibility to include the biofunctionalization process in the nano-structured material 10 fabrication without any additional step. This would provide a general biosurface using a very simple and elegant fabrication method, where any biotinylated compound could be anchored through streptavidin/biotin tandem.

To optimize the new material preparation, at first, the appropriate $15 \mathrm{SU}-8 / \mathrm{Phb}$ ratio was studied. Initial mixtures of SU-8 2000.5 and photobiotin solution $(100 \mu \mathrm{g} / \mathrm{mL})$ in DMSO were tested involving several $\mathrm{SU}-8 / \mathrm{Phb}$ volumetric ratios: 1/0.5, 1/1, 1/4, and $1 / 10$. These mixtures were spin coated on glass slides and photoresist crosslinking was performed as usual. For this 20 biotinylated material, XPS and contact angle measurements were done. Again, contact angle (Table 1) showed a decrease $\left(68^{\circ}\right)$ with regard to the non-modified SU-8 $\left(82^{\circ}\right)$ because of the lower hydrophobicity of the new surface. XPS global analysis also showed the presence of $\mathrm{N}$ and $\mathrm{S}$ atoms, indicating the biotin 25 incorporation to the SU-8 (Fig. 2a and 2b).

Table 1 Water contact angle values $\left(^{\circ}\right)$ for SU8/Phb mixed surfaces fabricated with different volume ratio.

\begin{tabular}{|c|c|c|c|c|}
\hline $\begin{array}{c}\text { SU8/Phb } \\
1 / 0(\mathrm{v} / \mathrm{v})\end{array}$ & $\begin{array}{c}\text { SU8/Phb } \\
1 / 0.5(\mathrm{v} / \mathrm{v})\end{array}$ & $\begin{array}{c}\text { SU8/Phb } \\
1 / 1(\mathrm{v} / \mathrm{v})\end{array}$ & $\begin{array}{c}\text { SU8/Phb } \\
1 / 4(\mathrm{v} / \mathrm{v})\end{array}$ & $\begin{array}{c}\text { SU8/Phb } \\
1 / 10(\mathrm{v} / \mathrm{v})\end{array}$ \\
\hline $80.3 \pm 0.8$ & $68.0 \pm 0.5$ & $64.0 \pm 0.2$ & $61.6 \pm 0.6$ & $58.2 \pm 0.3$ \\
\hline
\end{tabular}

30

Further, immunoassays with a-BSA and a-biotin were carried out, in a similar manner than onto biotin coated surfaces, to test the performance of this new technical procedure (Fig. 5). Quantitative results obtained are given in Table S3 (Suppl. Mat.), 35 where net signal, net signal/background ratio, and the standard deviation for a-biotin concentration employed in chips are compared. Net signal reached maximum and comparable values for SU-8/photobiotin $1 / 1$ and $1 / 0.5$ chips but signal/background ratio was much better for $1 / 1$ (background signal for $1 / 0.5$ chips 40 was too high even after OVA blocking). Samples corresponding to $1 / 4$ and $1 / 10$ did not provided better values. For $1 / 1$ chips, a calibration curve was performed. To this end, we tried to determine the biotin density on the surface. Thus, streptavidinATTO microarray with concentrations ranging from 0.05 to 100 ${ }_{45} \mu \mathrm{g} / \mathrm{mL}$ reacted specifically with the surface and the fluorescence intensity was measured before and after washing step. The first measurement was used for the calibration curve, and the second one to determine the amount of streptavidin remaining on the surface after the recognition. The highest values of fluorescence 50 after washings were achieved for $50 \mu \mathrm{g} / \mathrm{mL}$ streptavidin-ATTO. Taking into account the dimensions of the spot $(400 \mu \mathrm{m}$ diameter), a coating density of $0.772 \mathrm{pmol} / \mathrm{cm}^{2}$ of streptavidin was obtained, which means an immobilization yield of $10 \%$. This data is in agreement with other studies already reported in the 55 literature showing that when biotin-streptavidin-biotin bridge chemistry is used for DNA assembly, low surface coverage (e.g. $10 \%$ biotin residue) is appreciated and leads to the best results, while a larger amount of streptavidin adsorption does not lead to a larger amount of biotinylated-DNA binding. ${ }^{40}$ We concluded 60 that the best performances for the new biotinylated material were achieved employing polymerization of a SU-8/photobiotin solution 1/1 (v/v).

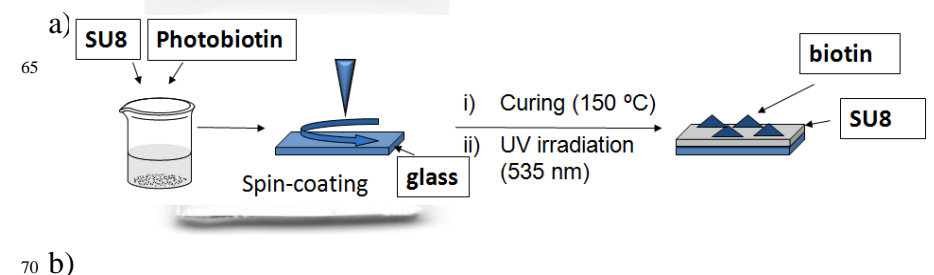

75

Fig. 5 (a) Scheme of biotinylation procedure incorporated to the SU-8 polymer fabrication. (b) a-BSA and a-biotin microarrays on SU-8/photobiotin 1/1 (v/v) chip. Concentration disposition is in 80 accordance with Fig. 4.

The new material was employed in the detection of the synthetic steroid gestrinone. It was used as a model system to demonstrate the applicability of the platform in screening assays such as 85 residue detection in sport doppping samples or pharmacological excretion residues. Specifically, a SU-8/Phb 1/1 (v/v) chip was used as assay platform and a competitive indirect format immunoassay was selected. Thus, the gestrinone hapten was conjugated to streptavidin (see Experimental), and the best 90 streptavidin-hapten concentration was identified and fixed at 996 $\mu \mathrm{g} / \mathrm{mL}$. After the blocking step, a microarray with a mixture of rabbit polyclonal sera solution (1/4000) and gestrinone at different concentrations $(0.2$ to $20 \mathrm{ng} / \mathrm{mL}$ ) was created (several replicates of each condition). Then GAR-Au was employed to 95 develop the array (see Experimental). Spots could be observed at naked eye. From the gestrinone dose-response curve (Fig. 6) the $\mathrm{IC}_{50}$ value was $1 \mathrm{ng} / \mathrm{mL}$, and the LOD was estimated at 0.26 $\mathrm{ng} / \mathrm{mL}$ of gestrinone. 
costs.

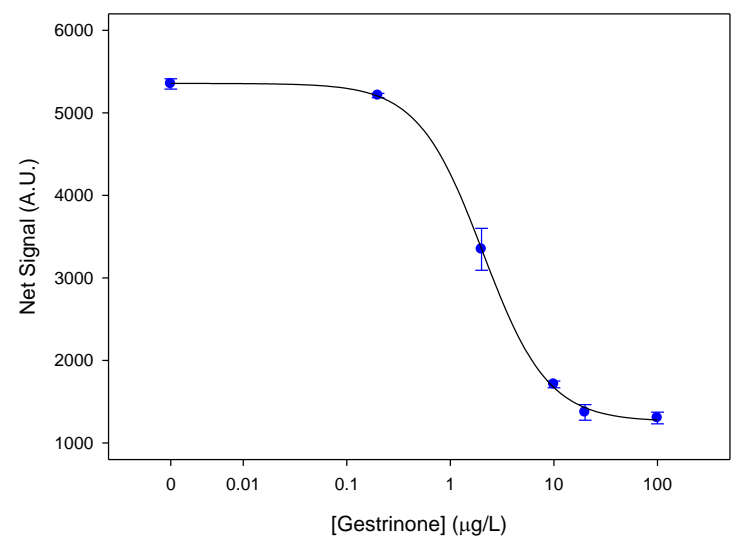

Fig. 6 Dose-response curve obtained for gestrinone competitive immunoassay.

It was demonstrated that the new biotin-ended material 5 obtained from reaction between SU-8 and photobiotin through coating or direct photopolymerization may be used to attach biotinylated probes, which can be further used to selectively detect targets by bioanalytical methods reaching high sensitivity. The potential of this new surface for the development of micro 10 and nanofabricated based biosensors is of high interest, as the own biofunctionalization process may be incorporated in the material fabrication for micro- and nano-structures, reducing the number of steps and associated cost. To prove this concept, arrays of nanopillars and nanostrips were fabricated using SU-8 and 15 with a
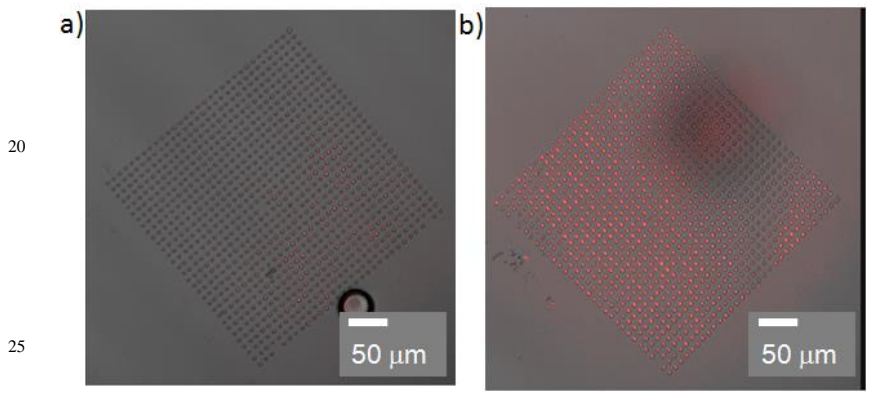

Fig. 7 Confocal image and fluorescence confocal image supperposed for an array of nanopillars fabricated in (a) SU-8 and 30 (b) SU-8/Phb 1/1 and incubated with ATTO-streptavidin for 10 minutes.

mixture of SU-8 and Phb (1/1 v/v ratio). The consistency of such structures was confirmed by confocal microscopy. The 35 bioavailability of biotin moieties on the nanopillars surface was demonstrated by incubating the chips, after the hydrolysis with sulfuric acid, with a solution of $50 \mu \mathrm{g} / \mathrm{mL}$ streptavidin-ATTO in PBS-T for 10 minutes. Fluorescence confocal microscopy images showed presence of stretavidin only on those structures fabricated 40 employing a mixture SU-8/Phb (Fig. 7). This assay demonstrated the suitability of the methodology to be employed for the construction of biosensors performing simultaneously the biofunctionalization and the structure fabrication, saving time and

\section{${ }_{45}$ Conclusions}

We have developed a new procedure for the chemical modification of SU-8 through the photobiotinylation promoted by UV radiation. The photobiotin coating of previously cured SU-8 surface was demonstrated, and its activity through different 50 bioaffinity assays was tested. Chemical behaviour of the new surface material was evaluated under distinct working conditions. Excellent results led to the development of a synthetic method for this novel material, which is based on the SU-8 curing process, and photobiotin mixture solution prepared with a range of volume 55 ratios and promoted by $350 \mathrm{~nm}$ radiation. This procedure simultaneously facilitates SU-8 polymerization and photobiotin incorporation to the polymer structure.

Immunorecognition assays showed excellent specificity and reproducibility, even when a competitive assay for the gestrinone 60 hormone determination was developed. Direct visualization of results with the naked eye is an advantage because it allows for immediate immunoassay monitoring without complex instrumentation. Furthermore, signal measurements can be performed with the assistance of a documental scanner, reaching 65 also a high sensitivity (LOD $0.26 \mathrm{ng} / \mathrm{mL}$ ) in a not fully optimized proof-of-concept immunoassay. The reached sensitivity compares with other well established immunoassay techniques as ELISA, where LOD of 0.09 and $0.14 \mathrm{ng} / \mathrm{mL}$ were obtained for gestrinone detection using direct and indirect ELISA plate formats 70 respectively. Therefore, this biosurface can be used for the immobilization of any biotinylated compound, while it is an interesting alternative to other materials in high-throughput biosensor development. The photopatterning of the surfaces has been demonstrated. Also, the inclussion of the biotinylation step 75 within the polymer fabrication proccess opens the possibility to employ it in the construction of low cost high density microarrays, ${ }^{41}$ as well as in the fabrication of integrated optical label free biosensors.

\section{Notes and references}

${ }_{80}{ }^{a}$ Centro de Reconocimiento Molecular y Desarrollo Tecnológico, Departamento de Química, Universitat Politècnica de València, Camino de Vera s/n, Valencia, Spain. Fax: +34 963879349 ; Tel:+34 963877340; E-mail: rpuchades@qim.upv.es

${ }^{b}$ Centro Láser, Universidad Politécnica de Madrid, Campus Sur, Madrid, 85 Spain.

${ }^{c}$ Instituto de Sistemas Optoelectrónicos y Microtecnología, Universidad Politécnica de Madrid, Ciudad Universitaria s/n, Madrid, Spain.

1 R. Narayanaswamy, O.S. Wolfbeis, in Optical Sensors, Springer, New York, United States 2004.

902 C. Liu, Adv. Mater., 2007, 19, 3783.

3 http://www.microchem.com/Prod-SU8_KMPR.htm.

4 B. Bêche, P. Papet, D. Debarnot, E. Gaviot, J. Zyss, F. PoucinEpaillard, Opt. Commun., 2005, 246, 25.

5 C.K. Chung, Y.Z. Hong, J. Micromech. Microeng., 2007, 17, 207.

956 H. Lorenz, M. Despont, N. Farhni, N. LaBianca, P. Renaud, P. Vettiger, J. Micromech. Microeng., 1997, 7, 121.

7 J.C. Ribeiro, G. Minas, P. Turmezei, R.F. Wolffenbuttel, J.H. Correia, Sens. Actuat. A-Phys., 2005, 123, 77.

8 T. Sikanen, S. Tuomikoski, R.A. Ketola, R. Kostiainen, S. Franssila, 100 T. Kotiaho, Lab Chip, 2005, 5, 888.

9 J. Lee, H. Shin, S. Kim, S. Hong, J. Chung, H. Park, J. Moon, Jpn. J. Appl. Phys. 2, 2003, 42, L1171. 
10 G. Genolet, M. Despong, P. Vettiger, D. Anselmetti, N.F. de Rooij, J. Vac. Sci. Technol. B, 2000, $18,617$.

11 T. Koerner, L. Brown, R.X. Xie, R.D. Oleschuk, Sens. Actuat. BChem., 2005, 107, 632.

512 A. Schneider, B. Su, T.W. Button, L. Singleton, O. Wilhelmi, S.E. Huq, P.D. Prewett, R.A. Lawes, Microsyst. Technol., 2002, 8, 88.

13 L. Jiang, K.P. Gerhardt, B. Myer, Y. Zohar, S. Pau, J. Microelectromech. S., 2008, 17, 1495.

14 D.A. Chang-Yen, B.K. Gale, Lab Chip, 2003, 3, 297.

1015 Y. Wang, M. Bachman, C.E. Sims, G.P. Li, N.L. Allbritton, Langmuir, 2006, 22, 2719.

16 R. Marie, S. Schmid, A. Johansson, L. Ejsing, M. Nordström, D. Häfliger, C.B.V. Christensen, A. Boisen, M. Dufva, Biosens. Bioelectron., 2006, 21, 1327.

1517 G. Blagoi, S. Keller, A. Johansson, A. Boisen, M. Dufva, Appl. Surf. Sci., 2008, 255, 2896.

18 M. Joshi, R. Pinto, R. Rao, S. Mukherji, Appl. Surf. Sci., 2007, 253, 3127.

19 M. Joshi, N. Kale, R. Lal, V.R. Rao, S. Mukherji, Biosens.

20 Bioelectron., 2007, 22, 2429.

20 A. Deepu, V.V.R. Sai, S. Mukherji, J. Mat. Sci.: Mater. Med., 2009, 20, S25.

21 K. Qvortrup, K.M. Taveras, O. Thastrup, T.E. Nielsen, Chem. Commun., 2011, 47, 1309.

2522 G. Cavalli, S. Banu, R.T. Ranasinghe, G.R. Broder, H.F.P. Martins, C. Neylon, H. Morgan, M. Bradley, P.L. Roach, J. Comb. Chem., 2007, 9, 462.

23 D. Sethi, A. Kumar, R.P. Gandhi, P. Kumar, K.C. Gupta, Bioconjugate Chem., 2010, 21, 1703.

3024 G.R. Broder, R.T. Ranasinghe, J.K. She, S. Banu, S.W. Birtwell, G.Cavalli, G.S. GAlitonov, D. Holmes, H.F.P. Martins, K.F. MacDonald, C. Neylon, N. Zheludev, P.L. Roach, H. Morgan, Anal. Chem., 2008, 80, 1902.

25 S.W. Birtwell, G.R. Broder, P.L. Roach, H. Morgan, Biomed. 35 Microdevices, 2012, 14, 651.

26 H.N. Kim, J.H. Kang, W.M. Jin, J.H. Moon, Soft Matter, 2011, 7, 2989.

27 B.Y. Shew, Y.C. Cheng, Y.H. Tsai, Sens. Actuators A, 2008, 141, 299.

${ }_{40} 28$ M. Holgado, C.A. Barrios, F.J. Ortega, F.J. Sanza, R. Casquel, M.F. Laguna, M.J. Bañuls, D. López-Romero, R. Puchades, A. Maquieira, Biosens. Bioelectron., 2010, 25, 2553.

29 F.J. Sanza, M. Holgado, F.J. Ortega, R. Casquel, D. López-Romero, M.J. Bañuls, M.F. Laguna, C.A. Barrios, R. Puchades, A. Maquieira,

45 Biosens. Bioelectron., 2011, 26, 4842.

30 F.J. Sanza, M.F. Laguna, R. Casquel, M. Holgado, C.A. Barrios, F.J. Ortega, D. López-Romero, J.J. García-Ballesteros, M.J. Bañuls, A. Maquieira, R. Puchades, Appl. Surf. Sci., 2011, 257, 5403.

31 F.J. Ortega, M.J. Bañuls, F.J. Sanza, R. Casquel, M.F. Laguna, M.

50 Holgado, D. López-Romero, C.A. Barrios, Á. Maquieira, R. Puchades, Biosensors, 2012, 2, 291.

32 S.A. Fleming, Tetrahedron, 1995, 51, 12479.

33 G. Blagoi, S. Keller, F. Persson, A. Boisen, M.H. Jakobsen, Langmuir, 2008, 24, 9929.

5534 M. Wilchek, E.A. Bayer, Anal. Biochem., 1988, 171, 1.

35 N. Dontha, W.B. Nowall, W.G. Kuhr, Anal. Chem., 1997, 69, 2619.

36 L.M. Wilde, G. Garace, C.J. Roberts, M.C. Davies, G.H. Sanders, S.J. Tendler, P.M. Williams, Analyst, 2001, 126, 195.

37 J.H. Choi, N.H. Kim, B.H. Chung, G.H. Seons, Anal. Biochem., $60 \quad 2005,347,60$.

38 E.M. Brun, A. Hernández-Albors, R. Ventura, R. Puchades, A. Maquieira, Talanta, 2010, 82, 1581.

39 M. Benlarbi, L.J. Blum, C.A. Marquette, Biosens. Bioelectron., 2012, 38, 220.

6540 K.M.M. Aung, X. Ho, X. Su, Sens. Actuat. B-Chem., 2008, 131, 371.

41 A. Malainou, P.S. Petrou, S.E. Kakabakos, E. Gogolides, A. Tserepi, Biosens. Bioelectron., 2012, 34, 273. 